\title{
The compassionate care: the divergences and the application limits in the relationship between risks and therapeutic benefits
}

\begin{abstract}
This article sets out the most important and critical issues related to the treatment of compassionate medicines through a reconstruction of the existing regulations in the Italian health system in comparison with that Spanish and American alike. The most important issue is the achievement of a balance between the risks arising from the use of drugs that have not completed the trial process with the benefits for the patient when there are no alternatives. This exhibition is interspersed with the indication of the paradigm cases of such treatments, the identification of good organizational models and a pilot project of mixed private participation, applied in the Italian health system. The final objective is to support the synthesis between present interests, decisionmaking participation and shared actions between the different stakeholders involved.
\end{abstract}

Volume 7 Issue 4 - 2019

\author{
Maria Carmen Agnello \\ Department of Law, University of Bologna, Italy
}

Correspondence: Maria Carmen Agnello, Department of Law, University of Bologna, Italy, Tel 095444225

3895749402, Email studio.mcagnello@libero.it

Received: May 06, 2019 | Published: July 09, 2019

Keywords: compassionate care, regulation, project public and private relationship, balancing risks, benefits

\section{Introductory aspects}

The compassionate care, an expression of Anglo-Saxon origin, indicates group therapies and for a single person in whom an experimental therapy is administered, not subject to institutional control and not yet marketed. These treatments concern the "niche" therapeutic areas related to "medical medical needs" that allow to treat rare diseases, patients in serious condition with poor therapeutic options, non responders, little studied in clinical trials. On the one hand medical activity cannot be exhausted with the ordinary prescription of medicinal products in the indications or methods of administration provided for by the marketing authorization. On the other hand, compassionate administration allows doctors and pharmaceutical companies to provide patients in serious health conditions with no other therapeutic options and not be able to enroll in a trial with medicines without authorization. The events related to the «compassionate use» of drugs have brought out the critical issues with respect to their administration (Stamina Case). The most critical profile regarding the administration of such drugs is to balance the protection of health in situations of extreme gravity and urgency with freedom of treatment in the dual sense of law "pretensive" as a choice, also of refusal to compassionate treatment.

The major issue involves assessing the relationship between risks and benefits, in prioritizing the well-being of the sick in relation to the interests of science and the socioeconomic. ${ }^{1}$ The necessary testing times, if for a verse require derogation from the process of experimentation and administration of drugs, on the other hand they cannot reduce safety standards, compared to what is recognized at a regulatory level for other pharmaceutical treatments. The "special use" of medicines for some diseases exposes the patient to potential

${ }^{1}$ National Committee for Bioethics, Case-by-Case Care and Invalidated Treatment (Compassionate Use) of 27 February 2015 p. 8-9, the Committee used the alternative expression "treatments not validated for personal and non-repeative use" for treatments not yet validated, even if they draw from a significant set of data in international scientific literature and scientific evidence, such that they are considered valid. risks, and as such implies correct information for the patient by the prescriber. Moreover, compassionate use concerns diseases, characterized by widespread diffusion, difficult placement and territorial delimitation (Ebola case), for which it is necessary as well as a clear regulation, able to overcome the application criticalities deriving from the normative stratification, also a discipline applicable to situations characterized by extraterritoriality.

These treatments have economic benefits for the health system and the patient, through the allocation of the economic burdens in the administration of these drugs to the manufacturing company

.$^{2}$ In this process, however, it is necessary to guarantee fairness and avoid inequalities by identifying clear assessment of the requirements for starting and accessing such treatments. These profiles have not only economic but also ethical implications, which in the writer's opinion constitute the most complex issues, with respect to incurable diseases and pathological situations characterized by unpredictable courses, where it is difficult to evaluate the appropriateness between tested therapies and between what is treatable outside of tried protocols.

Some cases concern Iressa, or gefitinibsidi administered compassionately as the benefit for non-small cell lung cancer has been demonstrated, but it has not concluded the institutional process of approving and marketing it. Another is Avastin, or bevacizumab, a drug belonging to the monoclonal antibody family which has been shown to be effective against metastatic colorectal cancer in trials. This drug, although approved in the European Union, but it will take time to be distributed in Italy, therefore the procedure for compassionate use has begun in order to administer it before the completion of the trial procedure. This article will analyse the compassionate use in the Italian health system through the lens of the primary objective to pursue health with respect to the interests of science and society,

${ }^{2}$ Investigation of the origin and development of the Stem Case of the 12th Permanent Commission (Hygiene and Health), 28 May 2014, www.parlamento. it, debate between bioethicists and lawyers on the relationship between the right to health and freedom of treatment highlights the aspects of difference and excludes the possibility of exclusive choice of the subject of the drug. 
through the regulatory framework and the role of A.I.F.A. in the management of pharmacovigilance. ${ }^{3}$

\section{The regulatory framework in a comparative view between health systems}

In view of the above, it is necessary to analyze the regulatory framework and the legal consequences, with respect to the behavior of healthcare professionals. ${ }^{4}$ For a correct, safe and effective use of drugs it is necessary to clarify both what are the regulatory limits within which "Special use" is allowed and what are the regulatory requirements underlying a correct administration in practice daily clinic. The birth of compassionate administration dates back to the mid-nineties, following the emergence of AIDS and Di Bella therapy. The goal was to mitigate the regulatory rigidity related to the prescription of these drugs, mediating between those who seek treatment in extreme situations and those who want to ensure effective and safe care. In this regard, the "International ethical guidelines for biomedical research" have identified the principles in the use of such drugs, such as "respect for the person", the "therapeutic" and "social" principle. $^{5}$

The question of the administration of these drugs has assumed legal importance with the art. 37 of the Helsinki Declaration and regulation 31 March 2004, n. 726, of the European Parliament and of the Council, which established Community procedures for the authorization and supervision of medicinal products for human and veterinary use. Article. 83 of EC Reg. N. 726/2004, II c. defines "compassionate use", the provision, for humanitarian reasons, of a medicinal product belonging to the categories referred to in art. 3, par. 1 and 2, to a group of patients suffering from a chronic or severely debilitating disease or considered to be life-threatening, without an alternative treatment with an authorized medicine. Pursuant to art 6 of the same, this medicine must be the subject of an application for marketing authorization and must be submitted as a subsequent clinical trial.

In Spain the royal decree n. 1015 of June 19, 2009 simplified the authorization process, in which the Spanish executive body regulates access to medicines without a therapeutic alternative and authorization, in a phase of clinical research in the face of serious health situations". Following a request presented online to the Spanish Medicines Agency, in coordination with other European agencies it temporarily authorizes such treatments to those who are in the same pathological situation. ${ }^{6}$ In this context, part of the doctrine highlighted

\footnotetext{
${ }^{3}$ National Committee on Bioethics, op. cit. according to which bioethics has identified in the stem case the right to hope", while in the United States laws have been enacted on the right to attempt"; (Right to try)in ensuring access to experimental drugs for terminally ill patients, without food and drug administration controls.

${ }^{4}$ L. pani and A. faia, ipazienti and the right to feel", true compassion or false hope? Controversial ethical aspects of Right to Trybill approved in some US States, of 30 June 2014, in www. AIFA.it, analyzes these profiles

${ }^{5}$ The International Ethical Guidelines for Biomedical Research, conducted on human beings", were published by the CIOMS (the Council of International Organisations and Medical Sciences), in 1993 in collaboration with the WHO, in Social Updates, 1994, n. 4, 1 e ss updated in 2002; U. accettella, Le radici della bioetica, edited by E. sgreccia, V. Mele, D. sacchini, Vol. II, Vita e Pensiero, 1998 , p. 8 and ss.

${ }^{6}$ Reg.(EC) No726/2004, guidelines on compassionate use of medicinal products, pursuant to article 83 of regulation (EC) No726/2004, EMA
}

the simplifying advantage of the authorization that allows a more extensive treatment, not limited to the individual case, avoiding further requests. ${ }^{7}$ However, doubts are raised in extending this model, as this procedure does not seem exhaustive in ensuring appropriateness, with the risk of implementing dangerous generalizations for treatments with high specificity.

In the United States, approval and faster access to "compassionate use" ("expanded access") drugs is permitted through "expanded access", experimental treatments, and those that are still being studied, after a case evaluation. to homes by the FDA to individuals suffering from a serious or life-threatening illness.

\section{The regulatory framework of the national health system}

In the Italian health system, the reference legislation is the Ministerial Decree of 8 May 2003, according to which, one can request a drug in a clinical trial phase in Italy or in another State from the manufacturing company for a non-experimental use on affected patients from serious or rare or life-threatening illnesses and in the absence of "valid therapeutic alternative". ${ }^{8}$ The therapeutic use of medicinal products subjected to clinical trials (so-called compassionate use) is defined as a free supply by the pharmaceutical company of medicines not yet authorized, subjected to clinical trials and produced in pharmaceutical plants or imported according to the authorization procedures and the requirements envisaged by current legislation; provided with the marketing authorization, pursuant to art. 6, paragraphs 1 and 2, of Legislative Decree 24 April 2006, n. 219 for indications other than those authorized, authorized but not yet available on the national territory. Pursuant to lett. e) the «expanded access program» therapeutic use program concerns the use of compassionate use drugs both in more patients, on the basis of a defined clinical protocol or on a nominal basis for a single patient, based on scientific and non-scientific evidence under a defined clinical protocol.

The use of the medicines referred to in art. 1 is required by the subjects referred to in art. 3, c.1, to the pharmaceutical company for the treatment of patients suffering from serious pathologies, rare diseases, rare tumors or in conditions of illness that put them in danger of life, for which valid therapeutic alternatives are not available or that do not may be included in a clinical trial or, for the purpose of therapeutic continuity, for patients already treated with clinical benefit as part of a completed clinical trial. The medicines referred to in art. 1 , c. 1, lett. a), must: be the subject, in the same specific therapeutic indication, of clinical trials, in progress or completed, of the third phase or, in particular cases of illness conditions that put the patient in danger of life, of clinical studies already concluded in the second phase. In guaranteeing safety it is necessary pursuant to letter. $b$ and c, the availability of data relating to the experiments pursuant to lett. a) sufficient to formulate a favorable judgment on the efficacy and tolerability of the medicinal product requested and be certified for production in accordance with good manufacturing practices (GMP).

The limit of the experiment is constituted, for rare diseases or /27170/2006.

${ }^{7}$ R. barranco Vela, The provision of medical services between right to health, principle of self-determination and optimal management of resources, by L. vandelli, C. bottari, M. Sesta, maggioli, 2014, cap. VI, par. 2.5, p. 184 s

${ }^{8}$ In G.U. n. 173 of 28 July 2003, modified by D.M. 7 November 2008 published in G.U. N. 80 of 6/4/2009. 
rare tumors, of medicines referred to in art. 1 with at least phase I experimental clinical trials, concluded and which have documented the activity and safety of the medicinal product, at a given dose and administration schedule, in indications also different from that for which compassionate use is required. In this case the possibility of obtaining a clinical benefit from the medicinal product must be reasonably founded on the basis of the mechanism of action and the dynamic drug effects of the medicinal product. The request for administration of such treatment, pursuant to art. 3 is presented by the doctor for a single patient not treated in the context of clinical studies, or in the context of therapeutic use programs, by several doctors operating in different centers or by multi-center collaborative groups, by the doctor or by collaborative groups for patients who have participated in a clinical trial that demonstrated a tolerability, safety and efficacy profile such as to constitute an indication of therapeutic continuity, even at the conclusion of the clinical trial. In the case of rare diseases and rare cancers, the request for medicines, submitted to phase I experimental clinical studies, is presented by the doctor who directs the clinical center identified by the region for the treatment of rare diseases or the clinical center belonging to the National Network of rare tumors.

To the D.M. of 2003 overlapped, without repealing it, the art. 12 of the "Balduzzi decree", entitled "Procedures concerning medicinal products", according to which "the application for classification of a medicinal product among those payable by the National Health Service could take place only after obtaining the marketing authorization (AIC) of the medicinal product and /or other drugs of exceptional therapeutic and social relevance envisaged in a specific resolution of the AIFA, or of medicines usable only in a hospital environment or in structures similar to it. In this case, the application can be submitted prior to the release of the marketing authorization (c. 2 and 3). Medicinal products for which a Community or national marketing authorization is issued, pending the submission by the company concerned of a possible application for a different classification, are placed in a special section, dedicated to drugs not yet assessed for reimbursement purposes and named by the Aifa Class C (nn), or Class C "non-negotiated" (c.5) ". 9 .

The legislation currently in force is more permissive than that of 2003, which admitted the request for the drug to the manufacturing company for use outside the clinical trial only when there is no valid therapeutic alternative to the treatment of serious diseases, or rare diseases or of life-threatening illness conditions and with the assumption of responsibility by the doctor and the patient.

Art. 2 of the D. Lgs. March 25, 2013, n. 24, amended upon conversion into Law 23 May 2013, n. 57, regulates the "medicines for advanced therapies prepared on a non-repetitive basis". In this regard, it is useful to carry out an analysis of the genesis of this legislation. ${ }^{10}$ The $\mathrm{c} .1$ of the art. 2 expected to administer such medicines exclusively in a public hospital, university clinic or hospital and scientific care,

${ }^{9}$ L. pani and A. faia, ipazienti and the right to feel", true compassion or false hope? Controversial ethical aspects of Right to Trybill approved in some US States, of 30 June 2014, in www. AIFA.it, analyzes these profile.

${ }^{10}$ According to art. 3, c. 1, lett. f-bis), Legislative Decree no. 219 of 2006, are those medicinal products "prepared on a non-repetitive basis, in accordance with specific quality requirements and used in a hospital, under the exclusive professional responsibility of a doctor, in execution of an individual medical prescription for a specific product intended for a particular patient", to which the general regime of Legislative Decree no. 219 of 2006 does not apply. "as the prescription was considered more efficient in the public structure. This arrangement, the expression of a favorlegisper for public structures, has been suppressed during the conversion, in avoiding discrimination for private structures and an unjustified limit by scientific evidence in administering such drugs. ${ }^{11}$

Instead, the art. 2 of the Legislative Decree n. 24 of 2013 entrusts the doctor with these prescriptive choices, without the comfort of scientific literature, in allowing a minimum of therapy in front of a poor prognosis and without care. This option, while allowing to protect health in extreme situations where it is necessary to avoid the excesses of "bureaucratisation", does not allow it to be applied whenever such drugs are required..$^{12}$ However, there are doubts about the appropriateness of such therapeutic treatments without the minimum requisites at the level of experimentation. In consideration of the above, the D.L. n. 24 of 2013, did not allow to guarantee a balanced management of scientific progress in this sector, where in art. 2 bases the administration of the drug on the autonomy of the doctor, who can pretend the technical-scientific results with respect to health protection. Furthermore, this discipline is lacking in the organization of the procedures, competences and responsibilities of the technical-scientific bodies involved, where it would have been necessary to coordinate the legal framework with scientific research.

From the launch oflawn. 38/2010 the management of compassionate use has evolved through the activities of the Regions that with the State-Regions Agreement of 16 December 2010 have launched the Guidelines for the coordination and development of regional

\begin{abstract}
${ }^{11}$ Besides, the c. 2 of the art. 2, what transitory norm, in the preceding version to the conversion it foresaw a further discrasia, in comparison to how much expressed to the c. The, in to allow the public structures in which I/you/ they have been on its way, anteriorly to the date of gone into effect of the decree, treatments on single patients with base medicines of cells staminali, even if prepared near laboratories you don't conform to the principles of the European norms of good manufacture of the medicines and in difformità of the dispositions of the D.M. of December 5 th 2006, you/they can complete the treatments themselves, under the responsibility of the medical prescrittore within the normative vigenteInoltre, the c. 2 of the art. 2, what transitory
\end{abstract}

norm, in the preceding version to the conversion it foresaw a further discrasia, in comparison to how much expressed to the c. The, in to allow the public structures in which I/you/they have been on its way, anteriorly to the date of gone into effect of the decree, treatments on single patients with base medicines of cells staminali, even if prepared near laboratories you don't conform to the principles of the European norms of good manufacture of the medicines and in difformità of the dispositions of the D.M. of December 5th 2006, you/they can complete the treatments themselves, under the responsibility of the medical prescrittore within the normative in force. Nevertheless, the disposition in to allow the treatments such conditions has not had a pacific application. The ord.. n. 414 of the 2012 of the TAR Brescia, have censored the profile of the formalities of carrying out of the experimentation, carente of one "methodic for the production and the therapeutic use of cells mesenchimali." P. White and others, of March 14th 2013, www.unipv-lawtech.eu has criticized in a letter to the Minister of the Health, "the use "compassionate", in single cases of therapies for which conclusive evidence of harmlessness and effectiveness don't exist you/he/she must not coincide with the ministerial authorization to put in to be in the structures sanitary therapies only supposed or unilaterally you define such"

${ }^{12}$ They are formulas endorsed by the majority doctrine, in P. cendon, stem cells administered to patients of serious neurological diseases, in Pers. and give, 2012, in favor of "treatments which appear dictated [... ] from consideration of pietas, solidarity, humility, charitable spirit, lack of alternatives, conjectural availability, mercy, therapeutic realism, comparative pragmatism, human understanding, in relation to the individual case". 
interventions in the area of the care network palliative. Subsequently, the agreement of 25 July 2012 set the minimum requirements and organizational methods necessary for the accreditation of terminal care facilities and palliative pain care units.

The regulatory framework has been enriched with the MD. 7 September 2017 on compassionate use, which proactively addressed the critical issues detected in the field of early and continuous access to new innovative drugs, in order to simplify administrative procedures and increase access to these drugs for rare diseases, while maintaining guarantees for patient safety. ${ }^{13}$ This regulation is not applicable to advanced therapy medicinal products, to which the D.M. health 16.01.2015, «Provisions on medicinal products for advanced therapies prepared on a non-repetitive basis».

The role of the ethics committee is to evaluate the application for the use of medicines pursuant to art. 1, previously submitted, by one of the doctors referred to in art. 3, accompanied by the clinical reason for the request, evaluating the relevant data relating to safety, tolerability and efficacy; patient information model; declaration of availability of the producer to the free supply of the medicine. In terms of safety, the dosage schedule and method of administration of which safety and activity are demonstrated in the clinical trials underlying the request will be evaluated. Furthermore, it is necessary to evaluate the degree of comparability of patients included in clinical trials and those for whom the request is made or, for rare diseases and tumors only, the existence of a common mechanism of action that makes a clinical benefit predictable in based on the available evidence for the medicine.

In light of the above, the "compassionate" use of drugs is subjected to stringent conditions dictated by the law and to clinical evaluation filters of the doctor that requires a supply in a detailed way and referred to a specific case to the pharmaceutical company. The authorizations of the hospital and patient ethics committee, which receives explanations on the pros and cons of the therapy and is required to sign an informed consent, are further guarantee measures. The drug must be previously inserted in a list prepared by the Single Commission of the Drug for compassionate use and must have passed the third and final phase. In some cases they concern Iressa, or gefitinibsi which has been shown to benefit non-small cell lung cancer, but has not concluded the institutional procedure that ratifies its approval and marketing. Another is Avastin, or bevacizumab, a drug belonging to the family of monoclonal antibodies which in the experiments proved effective against metastatic colorectal carcinoma; it was long awaited, the European Union approved it last January, but it will take time to be available, hoping to have it a year and a half earlier.

\section{The paradigmatic cases: treatment of "Bella" and "Stamina"}

Her above exposed normative antinomies I am expression of an intervention of the legislator disomogeneo and not always coherent in to face different stories from the treatment "Of Bella" to the case "Stamina" of which ripercorrere the more tappes it is necessary salient. Following the experimentation of the "you fine treatment Of Bella", the Constitutional Court in 1998, in to balance the right to the health with the liberty of care in safety terms, you/he/she has not recognized the right to get deprived pharmaceutical treatments of scientific base, but to a free treatment, in the ordinary experimentation

\footnotetext{
${ }^{13}$ G.U. 2 novembre 2017, n. 256.
}

what "medicinal innovative", if is "available results of clinical studies of second phase."

In another story related to the treatment produced by her/ it "Stamina Foundation", the sanitary system has recepito such care sustained by whole measures of urgency and normative. ${ }^{14}$ After a sanitary inspection, the AIFA has suspended the activity of laboratories, inosservanti the prescriptions of the art. 2 of the D.M. December 5th 2006, of "Use of medicines for therapy genica and for somatic cellular therapy out of clinical experimentations "edi treatments of therapy base jail cell of cells staminali, previous medical prescription. ${ }^{15}$ Despite this some families of smaller affections from illnesses neurodegenerative has gotten some provisions cautelari to continue the treatment near the public hospital that you/he/she had taken in load the patients. The same subjects, with the organization for the base treatment of cells staminali, has grasped the provision of the AIFA to the TAR Lombardia, of rejection the question of suspension to protect, in how much the test of effectiveness of the treatment doesn't satisfy the requisite" of scientific accreditation of the innovative therapeutic method. From the documentation and from the checks, in line to how much noticed by the AIFA, you/he/ she has not been possible to know the method of production and the therapeutic use of cells mesenchimali, recognized near the national and international scientific community". ${ }^{16}$

In view of this jurisdictional activity, the Government, by decree of urgency, has allowed such treatment to continue, with medicinal products produced by laboratories, by way of derogation from European standards of good manufacturing of medicinal products according to a treatment without any scientific literature, as contested by the pharmacovigilance authorities. By a subsequent decree of the Ministry of Health regulating the therapeutic use of medicinal products undergoing clinical trials, also with regard to medicinal products for advanced therapies", provision is made for "the supply of medicinal products free of charge by the manufacturer or the pharmaceutical company which proposed the trial", with the latter receiving the costs of such treatment. The discipline of compassionate access to care, in deregulating the prescription of drugs used in cell therapy, does not provide that the use of advanced therapy medicinal products should be based on a qualified index of "efficacy and tolerability". ${ }^{17}$ This

\footnotetext{
${ }^{14}$ TAR Lombardia, Brescia, ord. 5 .09. 2012, n. 414
}

${ }^{15}$ A. Abbott, stem-cell rulingriles researchers, in Nature, 2013,495, $418 \mathrm{sg}$. and N. Cartabellotta, Stem Cells: uncertainties of science and power of judges, in Pers. and damage, November 3, 2012.

${ }^{16}$ Reference is made to Regulatory capture, v. J.-J. laffont - J. Tirole, The politics of Government decision making: a theory of Regulatory Capture, in quart. Journ. of econ.,1991, Vol. 106, n. 4, 1089 sgg. ; G.J. STIGLER, The theory of Economic regulation, in The Bell Journ. of econ. andManag. Science, 1971, Vol. 2, n. 1, 10 ss In a different way than provided by'art. 1, c. 4, of D.L. n. 536 of 1996, which places the delivery of experimental drugs on SSN. There are differences between the two. The D.L. n. 536 of 1996 regulates drugs, for which there is a lack of an alternative therapeutic". This may not be the case for experimental advanced therapy medicinal products, although it is difficult for a doctor and a health establishment to undertake advanced therapy where there is a marketed alternative therapy. Moreover, the D.L. n. 536 of 1996 applies to medicinal products that have safety and efficacy indices, because placed on the market (even if for other therapeutic indications) or because they have been marketed in another State or because they have passed the second trial phase.

17 L'art. 13, c. 7,cod.deont. requires that "be scientifically documented", as well as l'art. Reg. 83 of the EC Reg. 31 March 2004, No 726 provides that the medicinal product for compassionate use "is to be the subject of an application 
is contrary to European law, which while allowing compassionate access to treatment through innovative therapies, which are not yet constitutive of medical practice, requires a reliable theoretical basis and experience. ${ }^{18}$

\section{The farmacovigilanza: the role of the to AIFA}

The AIFA develops an activity of farmacovigilanza regarding the effectiveness, to the subsistence of adverse events and tied up pharmacological interactions to such compassionate treatments through the harvest and diffusion of data, finalized to implement the informative level and the monitoraggio. Such activity is developed through the documentation trasmessaal proper Office Search and Clinical Experimentation, and in kind the application of the medicine formulated by the physician with assumption of responsibility to the treatment according to protocol to the senses of the art. $4 \mathrm{cs} .2$ letts. to) DM $8 / 5 / 2003$. The start of the programs of compassionate use in Italy is preceded by the preventive information to the AIFA from the pharmaceutical firms. Information concern the date of activation and closing of the program, the medicine to be made available in free form and the period of presumable availability to the free supply. They maintain the fattispecies regolatorie or safety cause of the interruption of office, in observance of the art. 83, c. 8, reg. n. 726/2004. You communication of closing of the program of compassionate use is to forward to the AIFA at least thirty days before the date of closing.

To the medicines for compassionate use the $\mathrm{D}$ is applied. M. health April 30th 2015, "operational Procedures and technical solutions for an effective action of farmacovigilanza adopted to the senses of the c. 344 arts. 1 L. 24.12.2012, n. 228 (1.stability 2013). Within the proper one 'activity, the physicians and other sanitary operators are kept to signal to the person responsible of farmacovigilanza of the sanitary structure of affiliation or directly to the national Net of farmacovigilanza through the portal of the AIFA and to the competent ethical Committee, the suspicious adverse reactions, specifying that it deals with treatment to compassionate use. Such signaling must be sends within two days and, for the medicines of biological origin not over the trentaseis times, in complete way and according to the formalities published on the site of the AIFA.

Subsequently, the person responsible of farmacovigilanza of the sanitary structure of affiliation of the signaller will notify the signaling to the AIFA and the firm fornitrice of the used medicine. The firm that has furnished the medicine is kept to manage the signaling of which to the c. 1 according to the anticipated formalities from the D.M. health April 30th 2015 and to inform the competent ethical Committee. ${ }^{19}$

The ethical Committee transmits to the Italian agency of the medicine (AIFA) his/her own opinion, equipped by the relative documentation, within three days from the adoption of the same opinion, for activity of monitoraggio on the different nominal uses and programs of compassionate use activated on the territory. The

for a marketing authorisation [...] or undergoing a clinical trial"; D.M. 8 May 2003, requires the drug to be medically tested; art. 3, c. 2, of D.L. n. 23 of 1998, containing"Urgent provisions concerning clinical trials in the field of oncology", inter alia, requires a "use [...] known and consistent with work published in scientific publications accredited in the international field

${ }^{18}$ This formula is by P. zatti, ideas on freedom of care: between experimentation and innovative therapy, in AA.VV., Studies in honor of P.Schlesinger, Vol. I, giuffré, Milan, 2004, 369 ss

${ }^{19}$ On the website of A.I.F.A. is published the List programs for compassionate use (update 3 April 2019). aifa, recognizes where the necessity of it for the guardianship of the public health, you/he/she can intervene in restrictive way and to suspend or to forbid the compassionate use of the signalled medicine.

Beyond the exposed run, the AIFA favors the dialogue proattivo with the interlocutors, in primis the citizens, through a comparison directed to address the activity regolatoria, in the priority interest of the patients. The acquired information and harvests and you publish through the Open Data related to the programs for compassionate use they constitute some lists of transparency through a list riepilogativo of the programs for compassionate (D.M May 8th 2003 - modified by the DM 7/11/2008) use. The updating of such list allows a constant monitoraggio of the use of the medicines, what information source on the prescriptive appropriateness and on the effectiveness of the therapies in the clinical practice. Besides, the AIFA is careful to the appeals of the stakeholders, promoters of runs that allow the access an innovative medicine, before the authorization. This happens with the application of insertion in the 648/1996 list, that allows the disbursement load of the National Sanitary Service, and you/he/ she can also be introduced from an association of patients or from a scientific society.

The picture shows that in such treatments it is necessary to support the patient experience and the know-how of the scientific community, as important factors in the decision-making process of the Agency. Following the agreement of $21 / 04 / 2015$, between AIFA and the European Medicines Agency a note has been issued regarding the compassionate use of nivolumab in II line and following for patients diagnosed with squamous cell lung cancer not resectable and/or metastatic.

In the light of the results of the Phase I, II, III clinical trials, AIFA initiated the compassionate use programme for nivolumab in line II and later, in order to ensure that patients diagnosed with non-removable and/or metastatic squamous lung cancer have timely access to effective pharmacological therapies. ${ }^{20}$ Based on the results of a specific therapeutic setting with poor prognosis and limited therapeutic alternatives, AIFA and the representative associations asked Bristol Myers to start a compassionate use program under the Ministerial Decree of 8 May 2003. The company was willing to activate the program, committing itself to accept requests for compassionate treatment until the European registration (EC decision).

\section{Pilot plans: partner's case ship between public and privacy}

A project pilot, develops in base the evidences and the patientfocused you/he/she has appraised her applications of compassionate use of the medicines, involving the manufacturing house and the Division of Etica Medica of the NYU Langone Medical Center. In 2015 the gruppoCompAC (you Sympathize with use AdvisoryCommittee), independent third subject formed by ten independent international experts, physicians, bioeticisti erappresentanti of the associations of the patients is compared suicriteri and on the procedures to answer to the necessities of the patient applicant the compassionate treatment and

\footnotetext{
${ }^{20}$ I risultati dello studio di fase III CheckMate-017 che confrontava, in pazienti con NSCLC squamocellulare metastatico in progressione, durante o dopo una I linea di chemioterapia contenente platino, il nivolumab vs. docetaxel, hanno mostrato un vantaggio di sopravvivenza altamente significativo, con OS mediana di 9.2 vs 6 mesi (HR=0,59 95\%IC 0.44,0.79; $\mathrm{p}=0,00025)$ a favore del trattamento con nivolumab.
} 
for the manufacturing firm of the in demand medicine. The objective is to guarantee the compassionate use of specific experimental medicines in development in observance of ethical principles through a transparent and equitable selective trial. Ilprincipale problem in the treatment with medicines for compassionate use I concerned limited lotteries produced for the clinical studies that could be carenti in the experimental iter.

The project pilots you/he/she has concerned the questions of access to the farmacodaratumumab, an antibody human monoclonale IgG1k that ties with high affinity ilrecettore CD38 expressed by the cells of the multiple mieloma. The product in the 2013 avevaricevuto from FDA the designation Fast Track Designation and Breakthrough Therapy, besides the status of orphan medicine, and you/he/she has been approved for the refractory multiple mieloma in the 2015. the ethical and procedural picture emerged by the jobs of the CompAC has appraised 76 applications for compassionate use, through the contact with the service of Medical Information USA or the sedelocale of the society for the other countries. The business site of recruitment for clinical glistudi has been optimized in base the indications delCompAC so that to improve the nformazione to the patients. The lines it drives for the physicians you are been implemented so that the question of access to the medicine develops him through standardized criterions. This is fundamental to guarantee that the following phase valutativa develops him in equitable way through equal information. The identity, the origin and the economic conditions are anonymous during the iter valutativo, in to guarantee a deprived evaluation of bias and to increase the trust of the patient and medical.

The medical manager and responsible of the progettoaziendale you/they have drawn the conclusions of the phase he/she pilots. The business attention toward the patient has been being confirmed for the maximum time of 5 days within which to appraise and to furnish an answer to the patients from the reception of the question. The evaluation from the CompAC is concluded with a recommendation to the firm, it has approvato60 questions; other two patients have been able to access the compassionate use of the daratumumab on decision of the firm. In case of refusal, the committee has decided to leave faculty to the taking care of physician to introduce an appeal furnishing documentation and support evidences of the question. The criterions of justice that have stamped the iter include, according to the article on JAMA, the not to damage the patient the probability to get an effective therapeutic answer, the functionality and the exhaustion of the other disposition approved treatments. With the purpose to assure the impartiality of the project in 2015, the applications for usocompassionevole for the experimental medicines are valued from an informed external committee, with the purpose to allow the best evaluation of these therapies in comparison to the condition of the patient and to the factors of risk, to take the decision most responsible."

This initiative, whose resulted I/you/they have been published in transparent way, you/he/she has wanted to create a model from guide to the decisions. Such model expressed by the project pilots on the daratumumab you/he/she has received the interest of associations and government institutions involved in the process of valutazionedelle questions of access to the cares for compassionate use. To such stregua, the application of such model could be wide, through the collaboration between the Janssen and the authority's regolatorie of different geographical areas. The programs of expanded access (EAP) could allow the access farmacisperimentali with a favorable profile risk-benefit proven by studies clinicipilota and for which a run is sustainable regolatorio up to the approval. In such picture, the patients with same conditions or in danger of life for which ilfarmaco has been studied, and that you/they have exhausted the other options of treatment, possonoaccedere to the program EAP. If, doesn't answer to the criterions of inclusionedell'EAP, the question for compassionate use can be late to the CompAC that guarantees the impartiality of the applications of access to the compassionate use of the experimental medicines, from that the pharmaceutical firms receive patient that they don't answer to other types of treatment anymore, they are not eligible for the share to the clinical trials in progress or to programs of expanded-access developed for specific groups of patients in accord with the authorities regolatorie or that they are by now under critical conditions.

\section{Final aspects: conflicts between ethical and therapeutics issues and balancing between benefits and risks}

To the light than exposed, in the pursuit of the priority objective of the comfort of the sick the pharmaceutical treatment 'compassionate' you/he/she cannot be considered what last choice but a valid pharmacological alternative of increasing therapeutic importance to be preferred in extreme situations, or rather when they are excluded or exhausted other treatments able to guarantee medicines efficaciin reduced times and without burdens to load of the patient

The decisions related to the compassionate use continue to be complex, in how much the actual system of assignment is still in work in progress. To such stregua, is necessary to individualize the runs with the purpose to implement the advantages and to reduce the risks for the health. The decision whether to approve or I lead an application for compassionate use, it would owe "to found on ethical and moral principles" more and more shared to level progettuale as express train from the site of the NYU School of Medicines.

Not the firms are always induced to cross the necessary iter for a new institutional approval and the physicians they are found to enter the circle of the so-called indications off-label, not suitable, neither from the firm neither from the office, but justified by the scientific literature. To such stregua, beyond the specific therapeutic finalities, the use "compassionate" introduces notable advantages for the patients to access the medicine to void costs, also through the help of the institution of a fund near the office of the Health.

For the firm manufacturing such platform of administration introduces a double advantage. The first one is to introduce the product on the market in swifter times without waiting for the anticipated times for the different degrees of experimentation. The second advantage is of the knowledge to make for the operators of the sector easier, preaddestrando the oncologis to the administration of a medicine in a therapeutic determinatacombinazione and for one determined pathology, object of studiche they also notice the effectiveness of it for other types of illness.

The therapeutic alternatives of complex application delimitation set to the professional operators of the matters of difficulty solution. For a verse, the discipline for the administration of the medicines to use c.d. compassionate (d.m 8 May 2003) it foresees the free supply to load of the pharmaceutical firm of a deprived medicine of authorization when valid therapeutic alternative doesn't exist to the treatment of serious pathologies or rare illnesses or of conditions of danger illness of life, for other verse, the art. 1, paragraph 4, d.1. October 21 st 1996, n. 536 foresee the supply to the patient, with burdens places in this case to load of the SSN of inserted medicines inun list (list 648), similarly if valid therapeutic alternative doesn't exist. 
In base to the normative in force, the prosecution of the cares for the experimental treatments in progress requires for both treatments the respect of the primary demand to guarantee the maximum level of guardianship to the right to the health and therapeutic safety.

At the base of such affairs and on the escort of the qualification of the discipline in theme of compassionate use as special, the routine is prevailing not to handle the classification in band $\mathrm{C}$, with the purpose to guarantee the continuity of access to the medicine in experimentation. Such more sustainable routine for the sanitary system is humiliated by the jurisprudence how prompt a careful monitoraggio of every procedure, so that is the experimentation that the compassionate use has effected with the necessary safety guarantees for the pazient. ${ }^{21}$ A case "paradigmatic" it concerns the chemio terapico CMF (ciclofosfamide, metotrexate and 5-fluorouracile), resulted effective in the care of the crab of the breast, even if this type of neoplasia is not suitable in the indications of the illustrative leaflet. In this case, the use is defined "precautionary", or possible solo within checked clinical studies or whereas there is not the presuppositions for the compassionate treatment.

The Role of the regional Institutions is to implement the function of guarantors in to protect the single consumers in relationship to the compassionate treatments avoiding the discriminations in the access and assignments to compassionate if possible eligibilities in clinical experimentation. To the respect, a paradigmatic case is the pharmacological Treatment for the hepatitis $\mathrm{C}$ for Patient sick with liver illness "advance "to which the are administered "lifesaver" or that they increase the effectiveness non available in commerce. The Region Lombardy has managed such situations through Lines it Drives and a Regional Decree n. 9982 of the 19.11.2015, that have introduced some idis crossed regional unified for access to the compassionate use and the therapeutic use of medicines submitted to experimentation (expanded access) with the purpose to guarantee a homogeneous management to territorial level of such treatments. Within such regulation of II I level to the public structures and the ethical committees it is up to adopt some protocols both to manage the routine and the urgency, with rapidity, efficiency, appropriateness, adequacy access to the cares.

The last part of this therapeutic circuit is the activity of clinicians and administrative staff to be implemented through the educational and organizational lever, oriented not only in the' perform such treatments quickly, but also to manage relations with patients and economic operators, which are seriously lacking in information in these sectors. The paths for the future are to change the current management oriented to management engagement, in order to increase user confidence in such treatments. This requires greater cooperation between health care stakeholders and joint efforts to address new therapeutic challenges and to coordinate individual interests in actions consistent with choices.

At the conclusion of the foregoing, it can be seen how compassionate care can give rise to conflicts of interest between subjects who are in different ways involved in the assitential circuit in the complex relationship between ethics, science and law, as expressed. Answer the question being asked. ${ }^{1}$ The conflict of interest between science and ethics arises when a contrast emerges whose overcoming implies

\footnotetext{
${ }^{21}$ The Council of State, opinion 14 November 2016, n. 2356, has answered the questions proposed by the Ministry of Health regarding the compatibility between the placement of a drug in C-grade, on those not yet assessed for reimbursement and c.d. compassionate use, in the case of diseases that endanger the patient's life and in the absence of a valid alternative therapy.
}

attributing to some requirements greater importance than others. The drama of some serious pathologies for which therapies already approved are not available often in situations of tension, sometimes of mutual opposition of rights, duties, hopes and expectations of each.

The observance of ethics implies not only to comply with the legislation even soft law, that is not binding, but also to respond to a plurality of needs through the predetermination of requirements pertaining to the activity to be implemented. These requirements also constitute assessment and control criteria, related to quality, understood as the social value and the scientific validity of such treatments and the observance of the principles of fairness of access and protection of the subject in guaranteeing a favorable relationship between risks. ${ }^{2}$ The ethical dilemma concerns the situations in which the non-authorization of a drug, which did not complete the entire procedure of apperation, would leave the patient without the only existing therapy, but at the same time the authorization of a cure, without data of complete safety would expose to risks not established.

In this way, such assessments as well as the resolution of ethical issues cannot be resolved only through regulation, but require an approach aimed at improving quality in health care, through American quality improvement initiatives or English and Italian clinical audit appropriateness studies. In particular, the scientific methodology followed was to formulate hypotheses that could be verified through predefined samples, in order to obtain valid results to be applied to similar cases. ${ }^{3}$ The Ethics Committee of the Emilia-Romagna Local Health Unit monitored the high density of requests for compassionate use of the drug avelimab, of which 86 in the $2015-2016$ period, in a reference population of the territory comprising 533,827 people, while between 2010-2015, with 610 out of 873,471 people. ${ }^{4}$ These requests were approved, as the high incidence of the request was shared by the ethics committees of other companies, the Hospital-University of Bologna-Policlinico S. Orsola-Malpighi. The above is an expression of a different path, in which the will of the institutions in decisions with respect to such care is oriented to "value for money", that is, to attribute a socio-welfare value as close as possible to the growing demand for these features.

\section{Acknowledgments}

None.

\section{Conflicts of interest}

Author declares that there is no conflict of interest.

\section{References}

1. Rosen CJ, Khosla S. Placebo-controlled trials in osteoporosis-proceeding with caution. $N$ Engl J Med. 2010;363(14):1365-1367.

2. Emanuel EJ, Wendler D, Grady C. What makes clinical research ethical? JAMA. 2000;283(20):2701-2711.

3. Murphy TF. Case studies in biomedical research ethics. Cambridge, Massachusetts: The MIT Press; 2004.

4. De Panfilis L, Satolli R, Costantini M. Compassionate use programs in Italy: ethical guidelines. BMC Med Ethics. 2018;19(1):22. 